\title{
Article
}

\section{Robotic Precisely Oocyte Blind Enucleation Method}

\author{
Xiangfei Zhao ${ }^{1,+}$, Maosheng Cui ${ }^{2,+}$, Yidi Zhang ${ }^{1}$, Yaowei Liu ${ }^{1, *}$ and Xin Zhao ${ }^{1}$ \\ 1 Institute of Robotics and Automatic Information System and the Tianjin Key Laboratory of Intelligent \\ Robotics, Nankai University, Tianjin 300071, China; 1120170124@mail.nankai.edu.cn (X.Z.); \\ yidi_zhang97@163.com (Y.Z.); zhaoxin@nankai.edu.cn (X.Z.) \\ 2 Institute of Animal Sciences, Tianjin 300112, China; tjxmcui2014@126.com \\ * Correspondence: liuyaowei@mail.nankai.edu.cn \\ + These authors contributed equally to this work.
}

check for updates

Citation: Zhao, X.; Cui, M.; Zhang, Y.; Liu, Y.; Zhao, X. Robotic Precisely Oocyte Blind Enucleation Method. Appl. Sci. 2021, 11, 1850. https://doi.org/10.3390/ app11041850

Academic Editors: Manuel Armada and Ioannis Kostavelis

Received: 30 December 2020

Accepted: 8 February 2021

Published: 19 February 2021

Publisher's Note: MDPI stays neutral with regard to jurisdictional claims in published maps and institutional affiliations.

Copyright: (c) 2021 by the authors. Licensee MDPI, Basel, Switzerland. This article is an open access article distributed under the terms and conditions of the Creative Commons Attribution (CC BY) license (https:// creativecommons.org/licenses/by/ $4.0 /)$.

\begin{abstract}
Oocyte enucleation is a critical procedure for somatic cell nuclear transfer. Yet, the main steps of oocyte enucleation are still manually operated, which presents several drawbacks such as low precision, high repetition error, and long training time for operators. For improving the operation efficiency and success rate, a robotic precise oocyte blind enucleation method is presented in this paper. The proposed method involves the following key techniques: oocyte translation control, oocyte immobilization and penetration control, and enucleation volume control based on the adaptive slide mode. Compared with the manual blind enucleation method, the proposed robotic blind enucleation method reduced the operation time by $44.5 \%$ (manual method: $62 \mathrm{~s}$ vs. proposed method: $34.4 \mathrm{~s}$ ), increased the accuracy of enucleation by $83.1 \%$ (manual method: $30.7 \mathrm{vs.}$. proposed method: 5.2), increased the success rate from $80 \%$ to $93.3 \%$, and increased the cleavage rate from $41.7 \%$ to $63.3 \%$.
\end{abstract}

Keywords: robotic blind enucleation; robotic cell manipulation; adaptive slide mode controls

\section{Introduction}

Cloning refers to the process in which cells develop into new organisms through asexual reproduction [1,2]. In 1997, British scientist Wilmut et al. used sheep mammary cells as donor cells for somatic cell nuclear transfer (SCNT) and obtained the world's first somatic cloned sheep, Dolly. Since the birth of Dolly, many other species, such as cloned mice, cloned dogs, cloned cows, cloned rabbits, cloned horses, etc., have also been born through nuclear transplantation technology [3-8]. The standard nuclear transplantation procedure is divided into two parts: the enucleation of oocytes and the injection of donor cells. Among them, the enucleation operation needs to tear apart the genetic material and cytoplasm in oocytes, which is the most difficult step in the operation of somatic cell nuclear transfer (SCNT). Yet, the main steps of oocyte enucleation are still manually operated [9], which presents several drawbacks, for example, low precision, high repetition error, and long training time for operators [10]. Therefore, it is necessary to automate the operation process of oocyte enucleation [11-13], which will improve the operation precision of oocyte enucleation, improve the success rate of oocyte enucleation, and further promote micro-manipulation technology and gene technology [14].

Recently, several studies have been carried out on the automation of oocyte enucleation. However, most of them automate the process by improving the hardware of the system, which will lead to higher cost and device incompatibility issues [15-17]. For example, Arai et al. designed and fabricated some new operating tools to fulfill the automatic enucleation process, including a magnetically driven micro-gripper for holding the oocyte and a magnetically driven micro-knife for rotating the oocyte and cutting the oocyte into half, then the part without genetic material was used as a somatic cell receptor [17-22]. However, this method removes too much cytoplasm, which will have an important impact on the developmental potential of oocytes [23,24]. Therefore, although this method is 
simple and easy to operate, it has not been widely used at present. The blind enucleation method is most widely used due to its advantages of low cost and relatively low amount of enucleation. It is necessary to design a robotic blind enucleation method, that is, only an enucleation micropipette (EM) and holding micropipette (HM) are used in the whole enucleation process.

In this paper, we developed a robotic precise oocyte blind enucleation method to precisely control the removed cytoplasm during enucleation and reduce whole operation time. Firstly, we designed the process of robotic enucleation. Secondly, we introduced the key techniques to solve the problems in the enucleation process, including the oocyte and micropipette detection method, oocyte translation control method, oocyte immobilization and penetration method, and enucleation volume control method. An adaptive slide mode controller (ASMC) was designed to generate enucleation accurately and the controller simulation was conducted to verify the controller's performance. Thirdly, we conducted the experiments of trajectory tracking for extracting cytoplasm. The control results show that the designed controller is able to track the trajectory and extract the specified cytoplasmic volume. Moreover, the proposed robotic enucleation method estimated the overshoot compared with the other control methods. Finally, we conducted the robotic enucleation experiments with porcine oocytes. The experimental results demonstrated that this robotic enucleation method is capable of enucleation with less time-cost, higher enucleation accuracy, higher enucleation success rate, and higher cleavage rate compared with the manual method.

The rest of the paper is organized as follows: Section 2 introduces the materials and methods of this paper, in which Section 2.1 introduces the process of robotic enucleation, Section 2.2 introduces the key techniques for robotic oocyte enucleation, and Section 2.3 introduces the controller simulation. Section 3 presents the experiments and the results, and Section 4 gives the conclusions of this paper.

\section{Materials and Methods}

\subsection{Process of Robotic Enucleation}

The process of robotic enucleation is shown in Figure 1. The detailed steps are listed as follows.

Step 1: Place oocytes into the medium culture before conducting the experiment.

Step 2: Move the motorized stage to the operation field until the oocyte to be operated on appears in the microscopic field.

Step 3: Translate the selected oocyte to the specified position in front of the HM. Then pick up the oocyte by reducing negative pressure to immobilize the embryo.

Step 4: Transfer the EM to the position close to the oocyte. Change the pose of the oocyte by the EM. The EM rotates the oocyte until the pole body appears at the desired position [25].

Step 5: The EM penetrates into the oocyte along the planned trajectory and extracts the specific volume of genetic material.

Step 6: Withdraw the EM from the oocyte and drain the genetic material out of the enucleation micropipette.

Step 7: Transfer the oocyte to the storage area and release it with positive pressure.

\subsection{Key Techniques}

\subsubsection{Oocyte and Micropipette Detection}

Figure 2 shows the process of image detection of the oocyte. The binary image is obtained by applying the Otsu adaptive thresholding method, as shown in Figure $2 \mathrm{~b}$. Based on the following morphological processing, the cytoplasmic binary region is shown in Figure 2c. The adaptive canny detection method was used to obtain the oocyte edge image, as shown in Figure 2d. After image closing and opening, the oocyte outline is obtained, as shown in Figure 2e,f. 


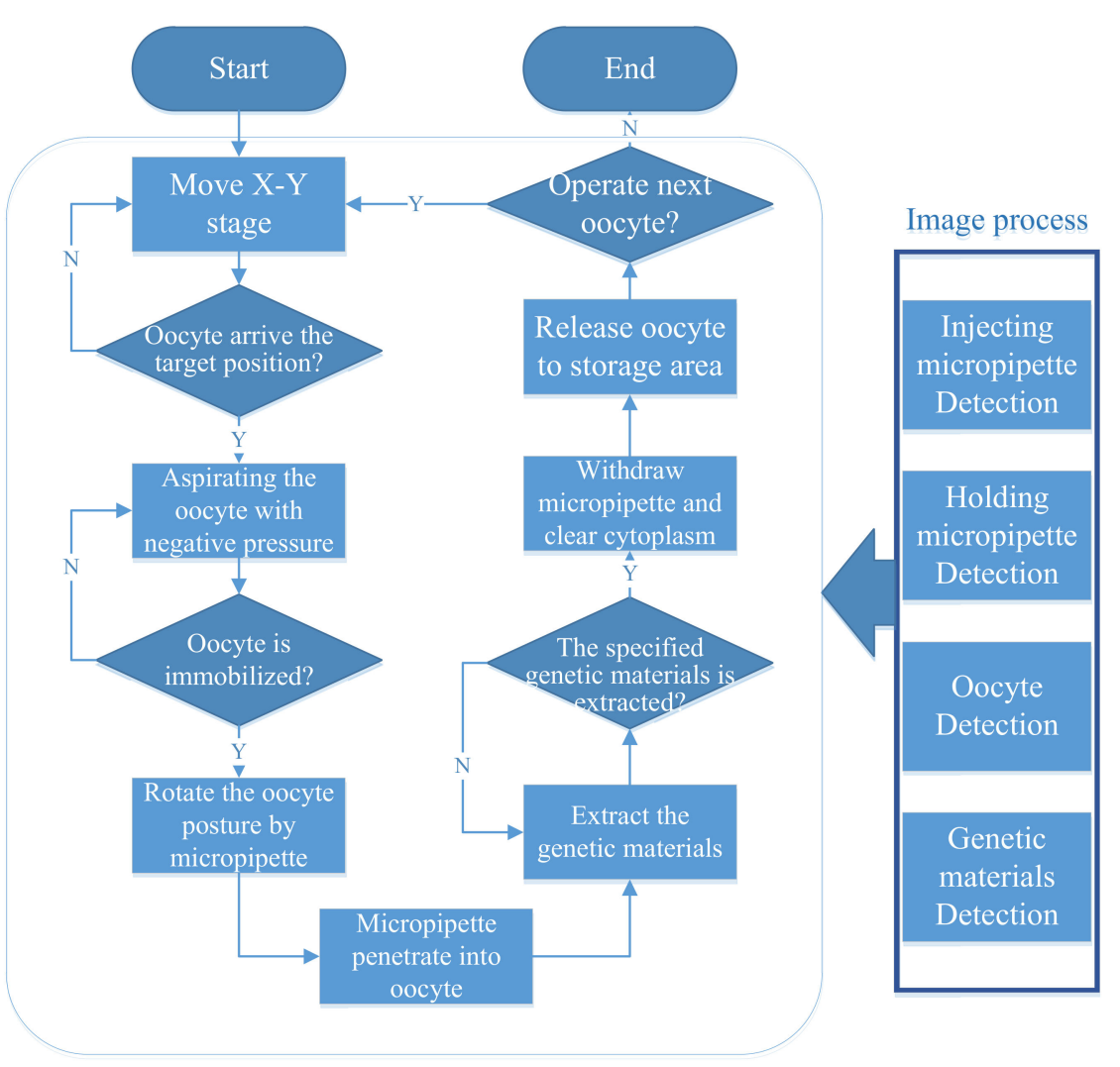

Figure 1. The process of robotic enucleation.
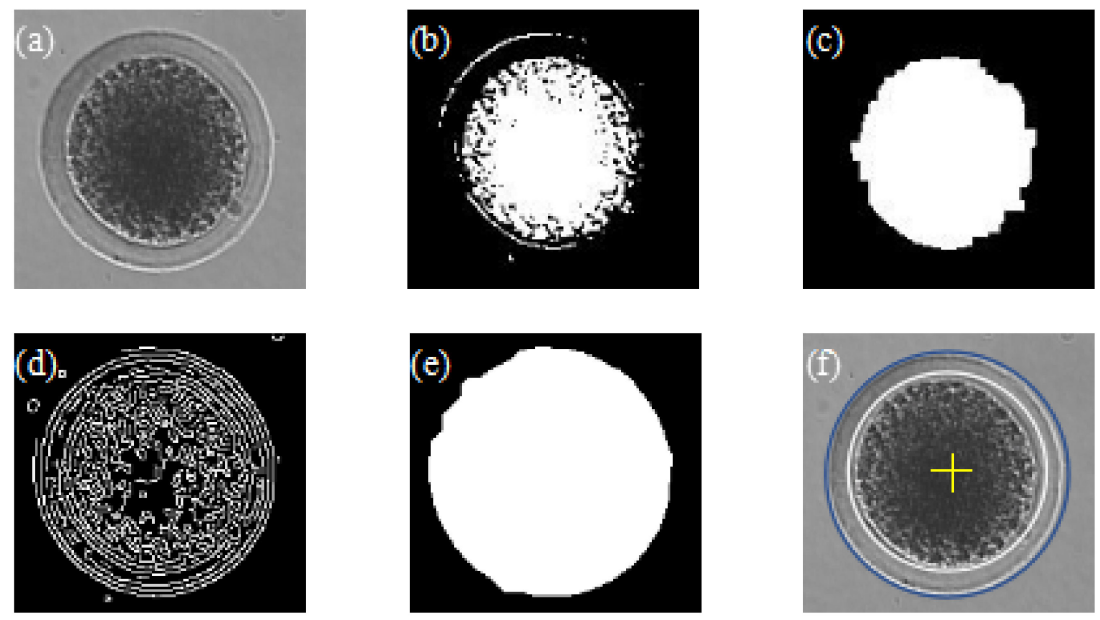

Figure 2. Oocyte detection. (a) Original image. (b) Binary image of the oocyte. (c) Image after morphological closing and opening. (d) Canny edge detection image. (e) Binary image after image closing and opening. (f) Oocyte detection result.

Figure 3 shows the process of image detection of the micropipette. The binary image is obtained by applying the Otsu adaptive thresholding method, followed by morphological processing, as shown in Figure 3b. The micropipette shape is reflected by the distance changes between the upper edge and lower edge of the micropipette. The micropipette tip area is detected by the distance changes, as shown in Figure 3c. The region next to the micropipette tip is defined as the cell tracking region, in which image processing is performed to track the length of genetic material. 


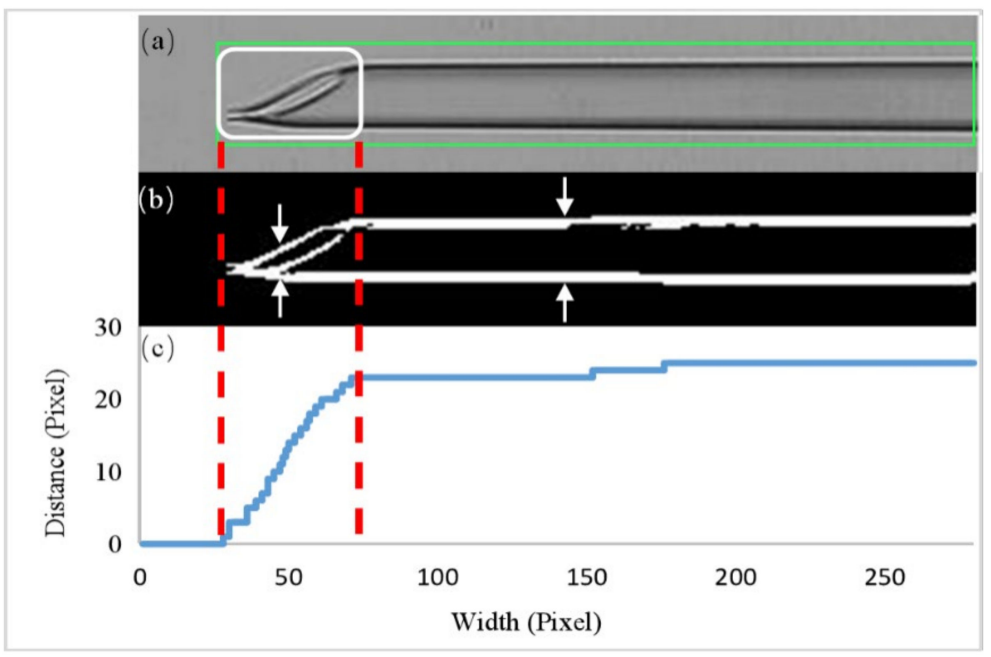

Figure 3. Micropipette detection. (a) Original image. (b) Binary image obtained by adaptive thresholding Otsu method. (c) The distance between the upper edge and lower edge of the micropipette.

\subsubsection{Oocyte Translation Control}

Oocytes are randomly distributed in the field of vision. The selected oocyte needs to be transferred to the specified position near the holding micropipette for immobilizing the oocyte, as shown in Figure 4. The petri dish is well placed on the motorized stage such that the motion of the oocyte and stage coincide with each other.

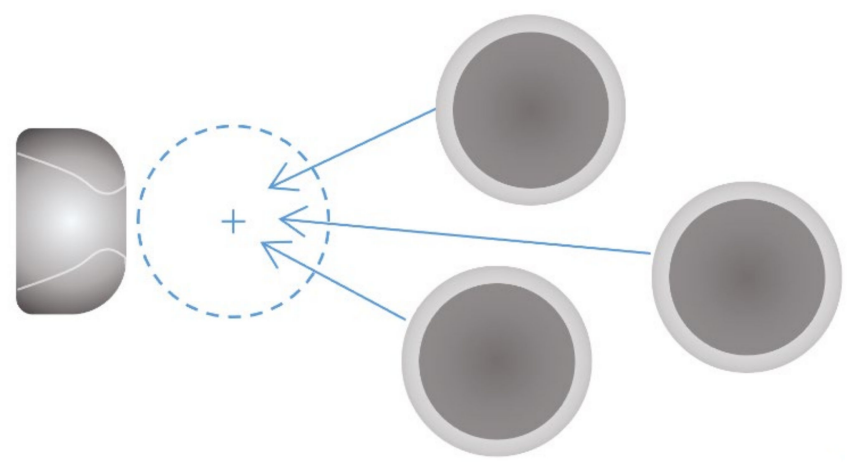

Figure 4. Schematic: Transferring the selected oocyte to the specified position near the holding micropipette for immobilizing.

The selected oocytes that transfer along the $X Y$ plane are seen as multiple agents in 2-D space. Therefore, there needs to be a motorized stage control loop based on the feedback of oocyte position. The transfer of multiple oocytes is sequentially performed by repeating the motorized stage control. The increment PID control is used to move the target oocyte to the specified position close to the HM. Different from the classic PID control, the incremental PID control makes a difference between the control quantity at the current moment and the control quantity at the last moment and takes the difference value as the new control quantity, which is a recursive algorithm.

Define position tracking error $e_{c}$ as:

$$
e_{c}=p_{d}-p(t)
$$

where $p_{d}$ and $p$ are desired trajectory and oocyte position, respectively. The incremental PID control is represented as follows:

$$
u(t)=u(t-T)+K_{p}\left[e_{\mathcal{c}}(t)-e_{\mathcal{c}}(t-T)\right]+K_{i} e_{\mathcal{c}}(t)+K_{d}\left[e_{\mathcal{c}}(t)-2 e_{\mathcal{c}}(t-T)+e_{\mathcal{c}}(t-2 T)\right]
$$


where $T$ is the sampling time interval, and $u(t-T)$ denotes the control variable in the previous time step. $K_{p}, K_{i}$, and $K_{d}$ are the positive gains to be designed.

\subsubsection{Oocyte Immobilization and Penetration}

The sharpness of the oocyte changes dramatically after immobilization, which can be treated as a criterion to detect whether the oocyte has been immobilized by the HM. The clarity evaluation method was employed to calculate the oocyte sharpness in the specific detection region. An evaluation function was defined by the normalized variance:

$$
F=\frac{1}{M \times N \times \varepsilon} \sum_{i=1}^{M} \sum_{j=1}^{N}[I(i, j)-\varepsilon]^{2}
$$

where $M$ and $N$ denote the height and width of the image, respectively. $I(i, j)$ is the pixel intensity at $(i, j)$, and $\varepsilon$ is the average intensity. Compared with the other clarity evaluation method [26], the normalized variance method provided a unimodal curve of the sharpness of the oocyte so that the focusing of the oocyte could be clearly distinguished.

The pneumatic syringe produced a negative pressure to aspirate the oocyte. At the same time, the evaluation function in the detected region was calculated in real-time. The cell immobilization state was determined by comparing the value of the evaluation function change in the detection region, as shown in Figure 5.
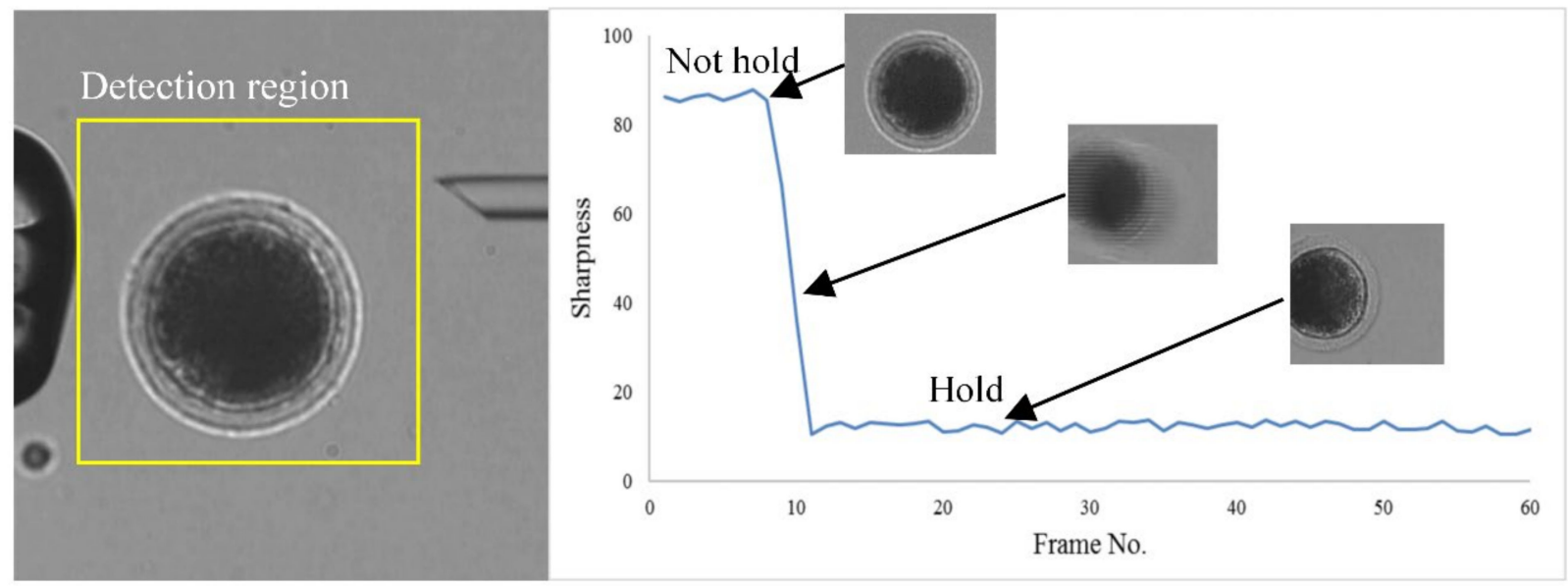

Figure 5. Sharpness of the detection region for cell immobilization detection.

After being held and rotated, the oocyte was penetrated by the EM for further enucleation. The EM movement was divided into three steps: (1) Move the EM from the starting point to the edge of the zona pellucida. (2) Penetrate into the oocyte along the $X$-axis. In the process of penetration, the penetration depth needs to be set to guarantee that the zona pellucida is punctured and damage to the oocyte is reduced as well. (3) Withdraw the EM to make the opening close to the polar body. A polar body is used as an enucleation target because the polar body is most likely next to the nucleus of the oocyte.

\subsubsection{Enucleation Volume Control Based on Adaptive Slide Mode}

To control the enucleation volume, the dynamics of the system need to be modeled to describe the cytoplasm motion inside the micropipette. In this paper, we used a model based on balance pressure that has been used in the cell microinjection [10,27]:

$$
m(x) \ddot{x}+c \dot{x}=\pi R^{2}\left(u-P_{c e l l}\right)-2 \pi \sigma \cos \beta R
$$

where the first item, $m(x) \ddot{x}$, is the mass of the substance inside the micropipette; the second term, $c \dot{x}$, is the resistance of the fluid, the third term, $\pi R^{2}\left(u-P_{\text {cell }}\right)$, is a composition of forces at the two ends of the micropipette, and the last term, $2 \pi R \sigma \cos \beta$, represents the 
capillary force. However, $c, \sigma$, and $\beta$ denote the damping of the culture medium, the surface tension coefficient of water, and the angle between the air-liquid interface and air-solid interface, respectively, and are treated as constants for a specific experiment; $m(x)$ is the material mass inside the micropipette, which is a time-varying item related to position $x$. $R$ and $P_{\text {cell }}$ denote the inner radius of the micropipette and the intracellular pressure [28], respectively; $u$ is the exerted external pressure, which is provided to the pneumatic pump by a stepping motor.

A certain amount of liquid exists in the micropipette before the cytoplasm is aspirated into the micropipette, and the liquid moves synchronously with the cytoplasm. Therefore, in the enucleation process, $m(x)$ should be divided into two parts: the mass of the medium culture $m_{0}$ and the mass of the cytoplasm $m_{\mathcal{c}}(x)$. Moreover, $m_{\mathcal{c}}(x)=\rho \pi R^{2} x$, where $\rho$ is the density of the cytoplasm. To facilitate the following description, we set $k=\pi R^{2}$, $\xi_{0}=2 \pi R \sigma \cos \beta$, then the dynamic model can be rewritten as:

$$
\begin{gathered}
\left(\rho k x+m_{0}\right) \ddot{x}+c \dot{x}+\xi_{0}+f=k u \\
f=-k P_{\text {cell }}
\end{gathered}
$$

where $f$ represents the lumped disturbance of the nominal system.

To overcome the effect of $f(t)$, the uncertainty and disturbance estimator (UDE) is used to estimate the term $f(t)$. Unlike other estimation techniques, UDE exhibits a suitable robustness against uncertainties, including the modeling error and parameter variation, and it is easy to implement in practice. The UDE is capable of estimating system disturbance by a low-pass filter when the signal bandwidth is appropriate. The estimated system disturbance is as follows:

$$
\hat{f}=\left[\left(\rho k x+m_{0}\right) \ddot{x}+c \dot{x}+\xi_{0}-k u\right] * g_{f}(t)
$$

where $*$ denotes the convolution operator and $g_{f}(t)$ is a filter involving $\lambda$ :

$$
g_{f}(t)=\mathcal{L}^{-1}\left\{\frac{(1-\lambda) \tau s+1}{\tau s+1}\right\}
$$

The estimation error of the lumped disturbance is defined as:

$$
|\widetilde{f}|=f-\hat{f}
$$

The following assumption is introduced:

Assumption: the estimation error of the disturbance is bounded, that is

$$
|\widetilde{f}| \leq p
$$

Controller Design and Stability Analysis.

As the cytoplasmic density $\rho$ and damping coefficient $c$ are unknown items, a slide mode adaptive controller is designed to control the volume of the enucleation cytoplasm.

First, the position error is defined as:

$$
e(t)=x(t)-x_{d}(t)
$$

where $x_{d}(t)$ and $x(t)$ are the desired and actual position trajectories, respectively.

We introduce the sliding mode variable $s$ :

$$
s=t e+\dot{e}
$$

where $t$ is a positive constant. 
Since the position error (11) converges to zero asymptotically when the parameters (cytoplasm density $\rho$ and damping coefficient $c$ ) within the system are unknown, an adaptive slide controller is designed as:

$$
u=\frac{1}{k}\left\{\hat{c} \dot{x}-\hat{\rho}\left[\frac{1}{2} k s \dot{x}+k x\left(t \dot{e}-\ddot{x}_{d}\right)\right]-\hat{f}+\zeta_{0}-m_{0}\left(t \dot{e}-\ddot{x}_{d}\right)-\eta \operatorname{sgn}(s)\right\}
$$

where $\hat{c}$ and $\hat{\rho}$ are the estimate values of $c$ and $\rho$, and the variable $\eta$ is the control gain.

The controller is generated through the following update law:

$$
\begin{gathered}
\dot{\hat{c}}=-\gamma_{2} s \dot{x} \\
\dot{\hat{\rho}}=-\gamma_{1}\left[\frac{1}{2} k s \dot{x}+k x s\left(t \dot{e}-\ddot{x}_{d}\right)\right]
\end{gathered}
$$

To demonstrate the stability of the designed controller, a Lyapunov function candidate is adopted as follows:

$$
V=\frac{1}{2}\left(\rho k x+m_{0}\right) s^{2}+\frac{1}{2 \gamma_{1}}(\rho-\hat{\rho})^{2}+\frac{1}{2 \gamma_{2}}(c-\hat{c})^{2}
$$

Differentiating Lyapunov function $V$ with respect to time gives:

$$
\begin{gathered}
\dot{V}=\frac{1}{2} \rho k \dot{x} s^{2}+\left(\rho k x+m_{0}\right) \dot{s}+\frac{1}{\gamma_{1}}(\rho-\hat{\rho})(-\dot{\hat{\rho}})+\frac{1}{\gamma_{2}}(c-\hat{c})(-\dot{\hat{c}}) \\
\dot{s}=t \dot{e}+\ddot{x}-\ddot{x}_{d}=t \dot{e}-\ddot{x}_{d}+\left(k u+f-c \dot{x}-\xi_{0}\right) /\left(\rho k x+m_{0}\right)
\end{gathered}
$$

Substituting (17) into (16) yields:

$$
\begin{gathered}
\dot{V}=\rho\left[\frac{1}{2} k \dot{x} s^{2}+k x s\left(t \dot{e}-\ddot{x}_{d}\right)\right]-s c \dot{x}+s f-s \xi_{0}+m_{0} s\left(t \dot{e}-\ddot{x}_{d}\right)+s k u \\
+\frac{1}{\gamma_{1}}(\rho-\hat{\rho})(-\dot{\hat{\rho}})+\frac{1}{\gamma_{2}}(c-\hat{c})(-\dot{\hat{c}})
\end{gathered}
$$

Substituting (13) into (19) yields:

$$
\dot{V}=-\eta|s|+s(f-\hat{f})+(c-\hat{c})\left(\frac{-\dot{\hat{c}}}{\gamma_{2}}-s \dot{x}\right)+(\rho-\hat{\rho})\left[\frac{-\dot{\hat{\rho}}}{\gamma_{1}}+\frac{1}{2} k \dot{x} s^{2}+k x s\left(t \dot{e}-\ddot{x}_{d}\right)\right]
$$

Substituting (14) and (15) into (20) yields:

$$
\dot{V}=-\eta|s|+s \widetilde{f}
$$

According to the assumption, to guarantee $\dot{V} \leq 0$, it is necessary to keep:

$$
\eta \geq h+p
$$

where $h$ is a positive constant. Then $\dot{V}$ satisfies

$$
\dot{V} \leq-\eta|s|
$$

which means that $\dot{V}$ is a negative definite.

Thus, we have $s \rightarrow 0$ and $e \rightarrow 0$ as $t \rightarrow 0$. The system is asymptotically stable.

\subsection{Controller Simulation}

In order to verify the controller's efficiencies before applying it to actual systems, simulation tests have been conducted to avoid possible damage to the system and oocyte. Specifically, the model and the controller are built in MATLAB/Simulink. The model parameters were chosen as $m_{0}=1 \mathrm{mg}$ and $r=10 \mu \mathrm{m}$. The control gains were chosen 
as $t=5.68, \tau=2$, and $\eta=3.5$. The ASMC parameter of update law was set as $\gamma_{1}=0.9$ and $\gamma_{2}=0.5$. The PID parameters are set as: $K_{p}=8.3, K_{i}=5.1$, and $K_{d}=2.8$. Figure 6 shows the ASMC simulation results compared with those obtained by a PID controller. The settling time and the tracking error for ASMC are less than PID. It can be seen from the control results that the PID controller has a large overshoot compared with ASMC. Since the cytoplasmic mass $\left(m_{c}\right)$ in the dynamic model is related to the cytoplasmic length $x$, the PID is difficult to compensate for the variation of cytoplasmic mass $m_{c}$. Therefore, the ASMC shows a better control performance than the PID in overshoot suppression and tracking accuracy.

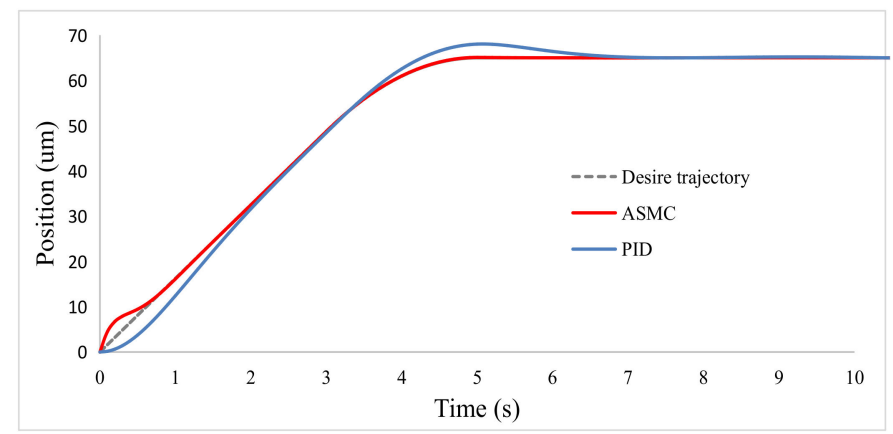

(a)

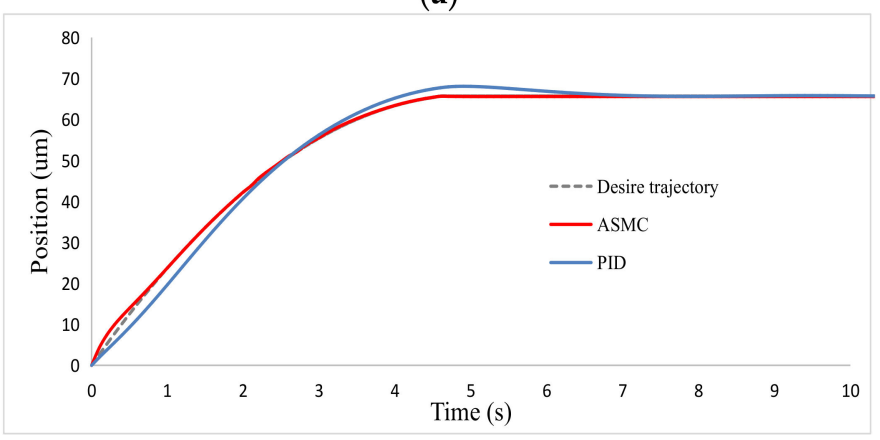

(b)

Figure 6. Controller simulation control results. (a) Ramp trajectory tracking control result. (b) Quadratic trajectory tracking control result.

\section{Results}

\subsection{System Setup}

The experiments were performed by the self-developed NK-MR601 micro-operation system [29-31]. Figure 7a shows the system setup, which is built based on a standard inverted microscope (CK-40, Olympus, Tokyo, Japan) equipped with a CCD (W-V-460, Panasonic, Osaka, Japan). The CCD was used to take microscope images with a resolution of $768 \times 576$ pixels, and the frame rate was 30 frame/s. The XY stage had a $30 \mathrm{~mm}$ travel range with $0.05 \mu \mathrm{m}$ resolution. A pair of $X Y Z$ micromanipulators (with a travel range of $50 \mathrm{~mm}$ and a maximum speed of $1 \mathrm{~mm} / \mathrm{s}$ ) were used for mounting the holding micropipette and the enucleation micropipette (EM), and a host computer was used for microscopic image processing and motion control of the $\mathrm{XY}$ stage, manipulators, and syringe pump. A pneumatic syringe (Narishige, IM-11B, Tokyo, Japan) was connected to the micropipette through a catheter. A stepping motor (Sanyo, 103H546-0410, Osaka, Japan) was connected with the lead screw of the pneumatic syringe through the coupling, and a syringe motor controller (Vince, VSMD101_025T, Beijing, China) was used to drive the step motor. The experiment was carried out in complete medium culture solution $(90 \% \mathrm{H} 199,10 \% \mathrm{FBS}, 1 \%$ CB). Porcine oocytes were used in the experiments. 

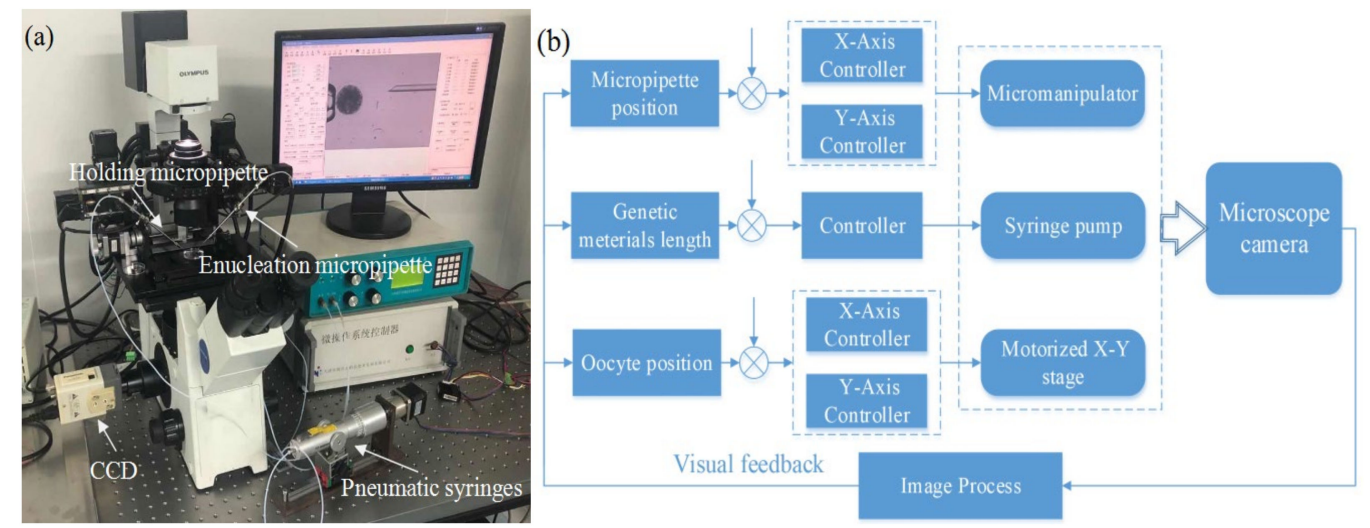

Figure 7. (a) NK-MR601 micro-operation system. (b) System control architecture.

Figure $7 \mathrm{~b}$ shows the system control architecture. The entire architecture is composed of three control loops. The positions of the oocyte, the length of the genetic material (GM), and the tip of the EM are used as visual feedback to develop visual servo control systems, respectively. The syringe pump, $X Y$ stage, and micromanipulator are controlled in coordination with the enucleation operation of the oocytes.

\subsection{Enucleation Micropipette Calibration}

The initial mass $m_{0}$ of the medium culture in the micropipette is calculated by $m_{0}=\rho_{m} \pi R^{2} L_{0}$, in which a common obstacle to measure the initial length of the medium culture $L_{0}$ is the skimpy field of view under a microscope, which usually causes the gas-liquid interface to move out of the field of view. To determine $L_{0}$, we calibrated the relationship between medium length $L$ and gas pressure $P$ based on the balance pressure model [27].

The liquid is aspirated into the micropipette by capillary pressure, ignoring the liquid gravity since the micropipette is placed horizontally. To quantify the influence of the capillary effect on aspiration pressure (capillary pressure), we provide a positive output pressure to offset the capillary force and stay the liquid in an equilibrium state. The relationship between medium length $L$ and gas pressure $P$ is calibrated, as shown in Figure 8.

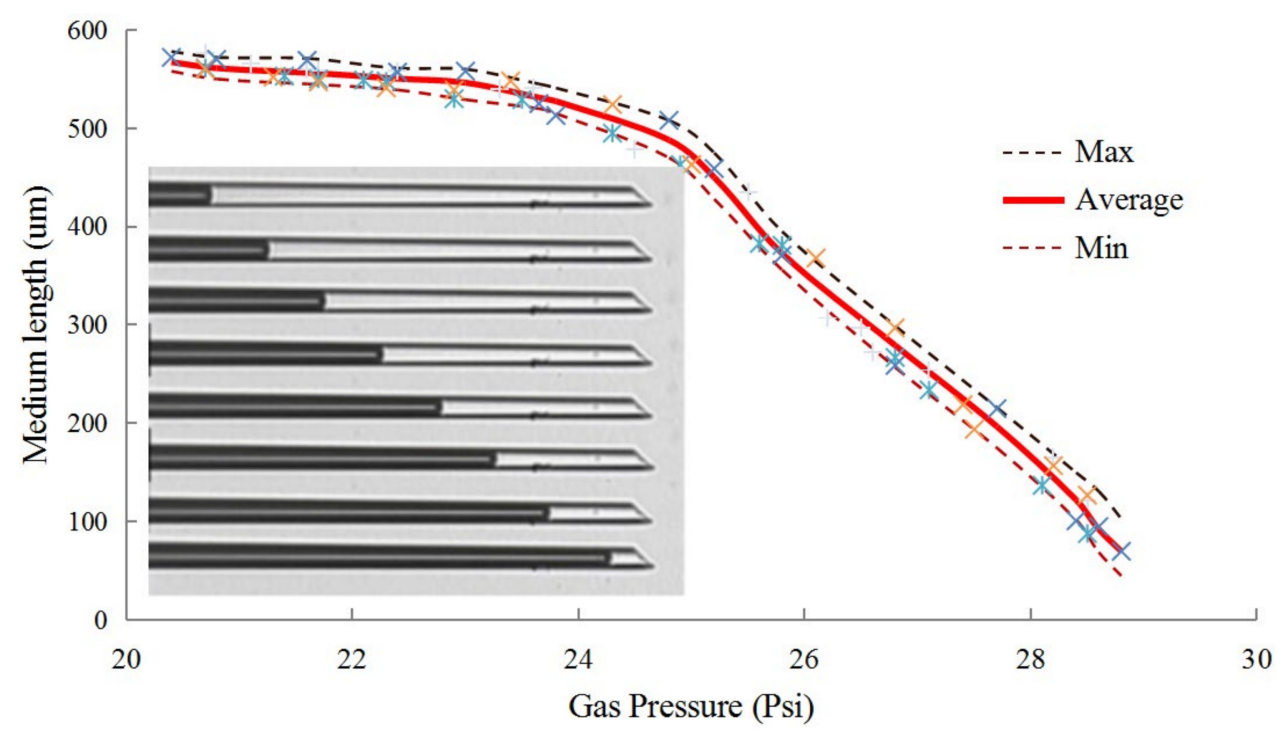

Figure 8. Calibration results between gas pressure and medium culture length. 


\subsection{Oocyte Translation and Enucleation Control Results}

Figure 9 shows the control results for translating the oocyte. The oocytes were scattered at the bottom of the petri dish. The target oocytes were initially located far from the HM. The PID controller was employed to control the selected oocyte to the HM. The control gains were $K_{p}=5.32, K_{i}=0.38$, and $K_{d}=1$. We used five porcine oocytes to verify the control method. The experimental results show that the oocytes reached the target position steadily without overshoot, and the translation time was less than $1.5 \mathrm{~s}$, which meets the requirements of the operation well.
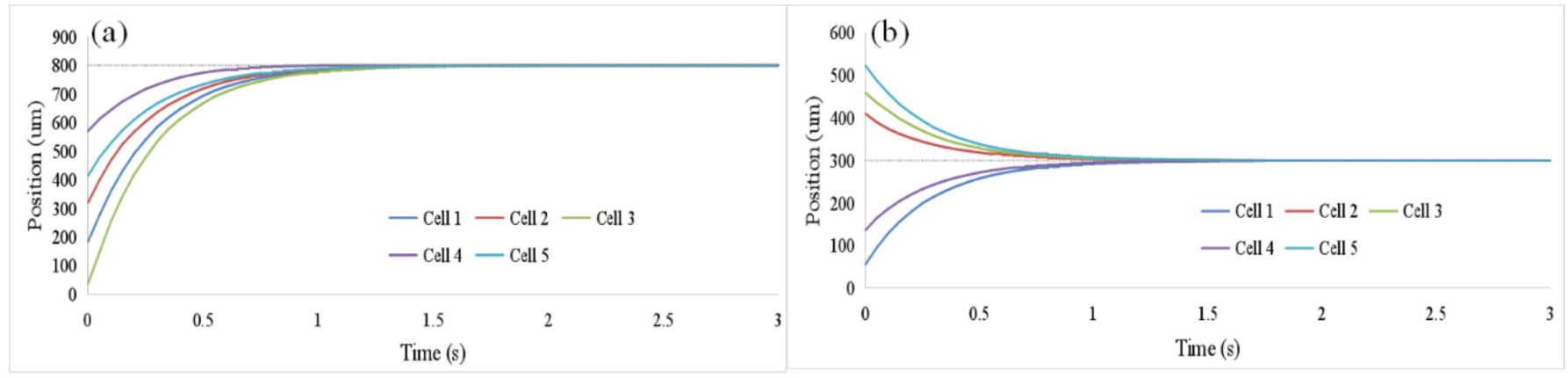

Figure 9. Oocyte translation control result. (a) $X$-axis direction control results. (b) $Y$-axis direction control results.

To evaluate the proposed controller performance, the enucleation experiments were carried out on different desired trajectories involving quadratic curves and ramps, respectively. The desired quadratic trajectory was determined according to the manual enucleation operation by an operation staff, where the actual trajectory of cytoplasm inside the micropipette was detected based on the background subtraction method (BSM). After data analysis, the trajectory could be well fitted with a quadratic polynomial. In addition, to better illustrate the performance of the proposed controller, we also used ramps as the desired trajectories in our experiment. The oocyte will reach the best enucleation results when the removed cytoplasm volume increases to $8 \%$ [32]. Thus, the enucleation target position inside the micropipette was determined by optimal cytoplasm volume. The quadratic trajectory was used as the desired trajectory in the enucleation experiments. Video (Movie.avi) given in the supplementary materials shows the control process of enu-cleation.

The porcine oocyte was used in the enucleation experiment, whose radius and density were $75 \pm 5 \mu \mathrm{m}$ and $1049.5 \pm 9.2 \mathrm{~kg} / \mathrm{m}^{3}$, respectively [33]. The density of the culture medium was $1008.2 \mathrm{~kg} / \mathrm{m}^{3}$ at $20^{\circ} \mathrm{C}$ [33]. The radius of the EM was $10 \mu \mathrm{m}$. The capillary force $\xi_{0}(t)$ is estimated by $\xi_{0}(t)=\pi R^{2} P(t) . P(t)$ is the measured gas pressure. The control gains were chosen as $t=3.74$ and $\eta=2.1$. The parameter of update law was set as $\gamma_{1}=0.5$ and $\gamma_{2}=2.1$. Figure 10 shows the control results for extracting the cytoplasm to its target position with two desired trajectories. To further demonstrate the superior performance of the designed control system, the experimental results were compared with those obtained by a PID controller. After sufficient tuning, the control parameters were set as: $K_{p}=13.2$, $K_{i}=1.8$, and $K_{d}=3$. Comparing with the PID control result, both tracking error and settling time on the ASMC were less than the PID. The PID have a large overshoot, which may result in large damage for the oocyte to affect the rate of survival. Therefore, the experimental results indicate the superior performance of the ASMC compared with the PID controller, in the sense of overshoot suppression and tracking accuracy, which facilitates the further application of enucleation. 


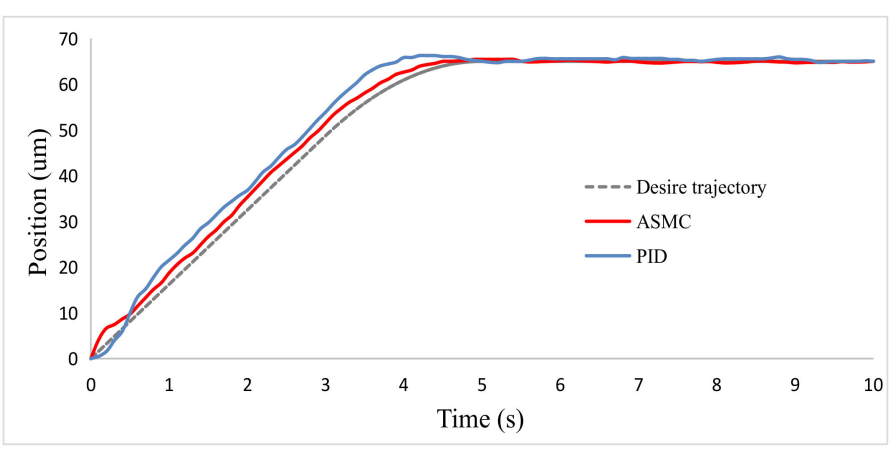

(a)

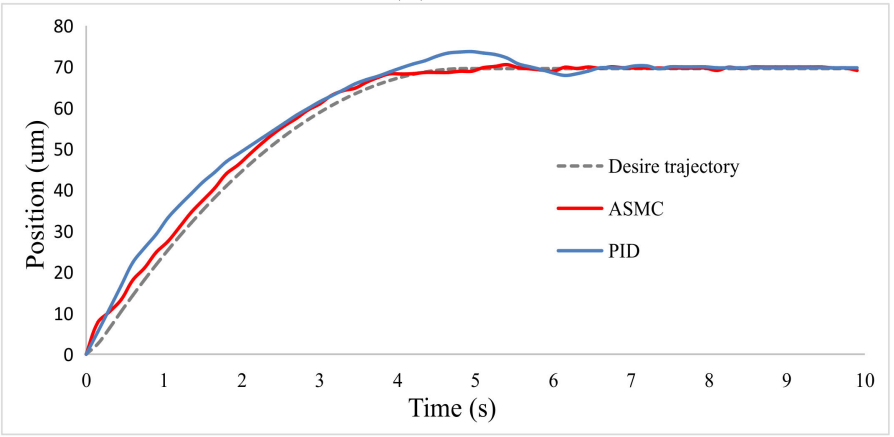

(b)

Figure 10. Cytoplasm extracting control result. (a) Quadratic trajectory tracking control result. (b) Ramp trajectory tracking control result.

\subsection{Enucleation Results}

A total of 30 oocytes were enucleated by the proposed robotic operation method. The typical operating process is shown in Figure 11. Figure 11a shows the process of translating the selected oocyte to the specified position near the HM. Figure $11 \mathrm{~b}$ shows the process of reducing pressure to immobilize the oocyte. Figure 11c shows the process of rotating the oocyte. Figure 11d shows the process of penetrating the EM into the oocyte following the planned trajectory. Figure 11e shows the process of aspirating the specific volume of cytoplasm and genetic material by the proposed SMAC method. Figure 11f shows the process of withdrawing the EM from the oocyte and releasing the enucleated cytoplasm and genetic material.
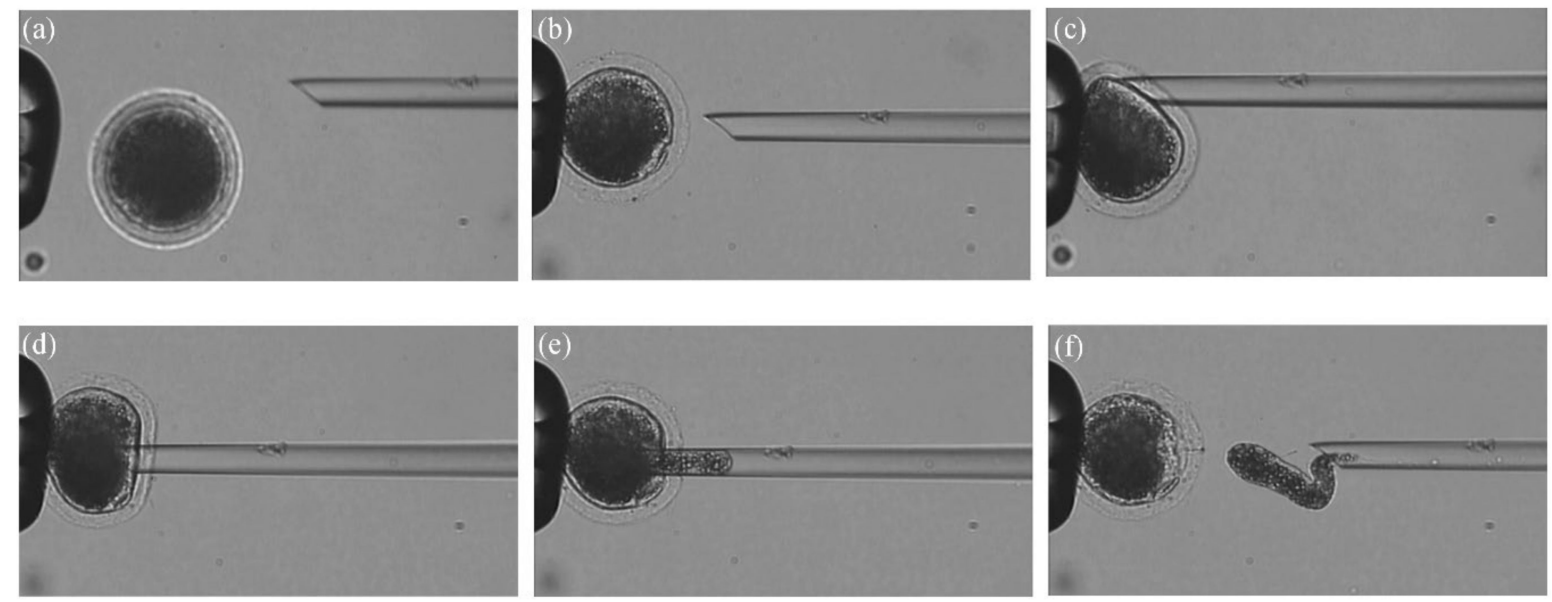

Figure 11. Oocyte enucleation operating process. (a) Translate selected oocyte to the specified position near the HM. (b) Reduce pressure to immobilize the oocyte. (c) Rotate the oocyte. (d) Penetrate the EM into the oocyte following the planned trajectory. (e) Aspirate the specific volume of cytoplasm by the proposed SMAC. (f) Withdraw the EM from the oocyte and remove the cytoplasm. 
In order to validate the effectiveness of the proposed enucleation method, the average enucleation time, enucleation accuracy, enucleation success rate, and the developmental potential after enucleation were counted and compared with those obtained in the manual blind aspiration method. The results are summarized in Table 1.

Table 1. Operation comparison between the manual and proposed enucleation methods.

\begin{tabular}{ccccc}
\hline Method & Average Time (s) & $\begin{array}{c}\text { Accuracy } \\
\text { (Standard Deviation) }\end{array}$ & Success Rate & Cleavage Rate \\
\hline Manual method & 62 & 30.7 & $80 \%$ & $41.7 \%$ \\
Proposed method & 34.4 & 5.2 & $93.3 \%$ & $63.3 \%$ \\
\hline
\end{tabular}

From translating oocytes to the extraction of genetic material, the average costing time for the proposed method was $34.4 \mathrm{~s}(n=30)$, which was much smaller than the manual method (62 s). The maximum time consumed in operating on a cell was $52.8 \mathrm{~s}$, and the minimum was $14.2 \mathrm{~s}$. The reason for the large operation time difference is that the posture of each immobilized oocyte is random, so the rotated angle and the time consumed are different between each other.

In the process of enucleation, the volume of cytoplasm inside the EM is fed back to the control system by image processing. The desired quadratic trajectory was tracked by the designed adaptive slide mode controller. Based on the fact that the removed cytoplasm and genetic material will form into a sphere in the culture medium, we counted the volume of enucleation, as shown in Figure 12a. The volume of enucleation was $8 \%$ of the cytoplasm. We calculated the standard deviation of the enucleation volume as the criterion of enucleation accuracy. The result shows that the accuracy of the proposed enucleation method was 5.2, and the accuracy of the manual method was 30.7. Then, we counted the success rate of enucleation by the oocyte fluorescent dye method. The results demonstrate that the success rate was $93.3 \%(28 / 30)$ by the proposed enucleation method. The accuracy of enucleation and success rate of enucleation are both significantly improved in comparison to the manual blind enucleation method [34], as shown in Table 1.
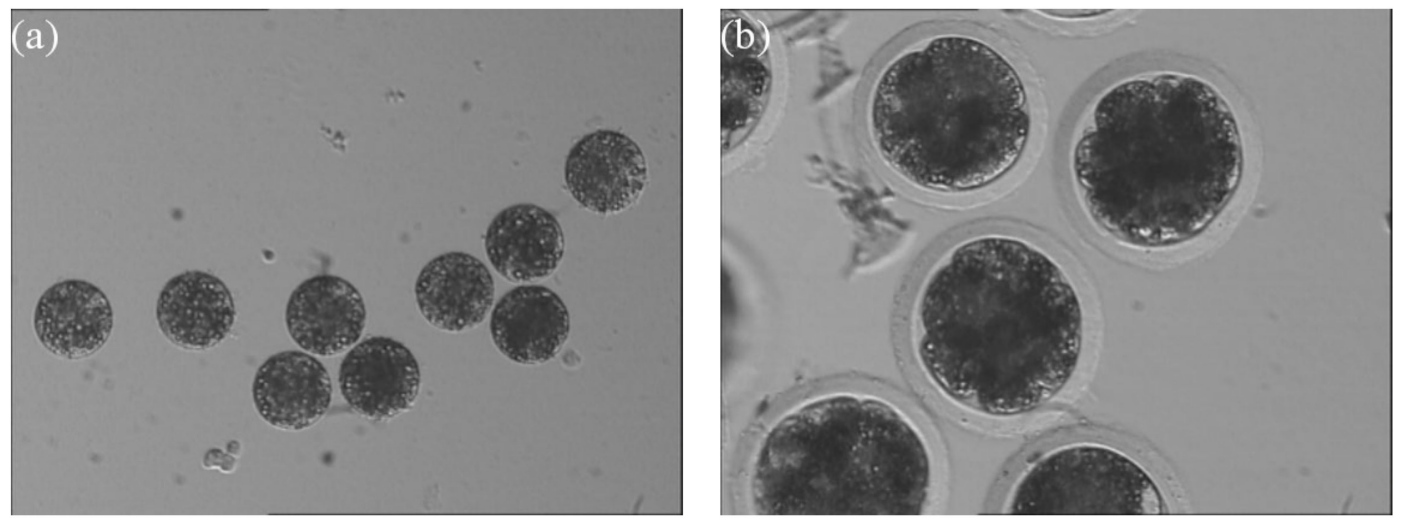

Figure 12. Enucleation results. (a) The removed cytoplasm and genetic material formed into spheres in the culture medium. (b) Embryos after oocyte enucleation, somatic cell injection, and cultured for $24 \mathrm{~h}$.

At last, we injected the somatic cell into the enucleated oocyte and recorded the cleavage rate of the embryos. The cleavage rate was used to qualify the developmental potential after enucleation. The embryos after enucleation and somatic cell injection were cultured at $37^{\circ} \mathrm{C}$ with $5 \% \mathrm{CO}_{2}$ for $24 \mathrm{~h}$, as shown in Figure $12 \mathrm{~b}$. The cleavage rate after enucleation with the proposed method was $63.3 \%$ (19/30), which was also significantly improved in comparison to the manual blind enucleation method (41.67\%) [32]. The low time-cost, high accuracy, high success rate, and high cleavage rate could demonstrate the effectiveness of the proposed robotic enucleation method. 


\section{Conclusions}

In this paper, we developed a robotic oocyte enucleation method. Firstly, we designed the process of robotic enucleation. Secondly, we introduced the key techniques to solve the problems in the enucleation process, including the oocyte and micropipette detection method, the oocyte translation control method, the oocyte immobilization and penetration method, and the enucleation volume control method. Thirdly, we conducted the experiments of trajectory tracking for extracting the cytoplasm. The control results show that the designed controller was able to track the trajectory and extract the specified cytoplasmic volume. Moreover, the proposed robotic enucleation method estimated the overshoot compared with the other control methods. Finally, we conducted the robotic enucleation experiments with porcine oocytes. The experimental results demonstrate that this system is capable of reducing the operation time by $44.5 \%$ (manual method: $62 \mathrm{~s}$ vs. proposed method: $34.4 \mathrm{~s}$ ), increasing the accuracy of enucleation by $83.1 \%$ (manual method: 30.7 vs. proposed method: 5.2 ), increasing the success rate from $80 \%$ to $93.3 \%$, and increasing the cleavage rate from $41.7 \%$ to $63.3 \%$. The low time-cost, high enucleation accuracy, high enucleation success rate, and high cleavage rate could demonstrate the effectiveness of the proposed robotic enucleation method.

Supplementary Materials: The following are available online at https:/ / www.mdpi.com/2076-341 $7 / 11 / 4 / 1850 /$ s1.

Author Contributions: X.Z. (Xiangfei Zhao), Y.L. and X.Z. (Xin Zhao) conceived the idea for the study. X.Z. (Xiangfei Zhao), Y.L. and M.C. designed the experiments. X.Z. (Xiangfei Zhao), Y.Z. and Y.L. performed the experiments. X.Z. (Xin Zhao) supervised the project. X.Z. (Xiangfei Zhao) and Y.L. wrote the manuscript. All authors have read and agreed to the published version of the manuscript.

Funding: This research was supported by the National Key R\&D Program of China (2020YFB1313101), National Natural Science Foundation of China (62003174, U1813210, 62003173) and China Postdoctoral Science Foundation (2020M680865).

Institutional Review Board Statement: Not applicable.

Informed Consent Statement: Not applicable.

Data Availability Statement: The data used to support the findings of this study are available from the corresponding author upon request.

Conflicts of Interest: The authors declare no conflict of interest.

\section{References}

1. Wilmut, I.; Beaujean, N.; De Sousa, P.A.; Dinnyes, A.; King, T.J.; Paterson, L.A.; Wells, D.N.; Young, L.E. Somatic cell nuclear transfer. Nature 2002, 419, 583-587. [CrossRef]

2. Wilmut, I.; Schnieke, A.E.; McWhir, J.; Kind, A.J.; Campbell, K.H.S. Viable offspring derived from fetal and adult mammalian cells. Nature 1997, 385, 810-813. [CrossRef]

3. Cibelli, J.B.; Stice, S.L.; Golueke, P.J.; Kane, J.J.; Jerry, J.; Blackwell, C.; De León, F.A.P.; Robl, J.M. Cloned Transgenic Calves Produced from Nonquiescent Fetal Fibroblasts. Science 1998, 280, 1256-1258. [CrossRef] [PubMed]

4. Wakayama, T.; Perry, A.C.F.; Zuccotti, M.; Johnson, K.R.; Yanagimachi, R. Full-term development of mice from enucleated oocytes injected with cumulus cell nuclei. Nature 1998, 394, 369-374. [CrossRef]

5. Chesné, P.; Adenot, P.G.; Viglietta, C.; Baratte, M.; Boulanger, L.; Renard, J.-P. Cloned rabbits produced by nuclear transfer from adult somatic cells. Nat. Biotechnol. 2002, 20, 366-369. [CrossRef]

6. Galli, C.; Lagutina, I.; Crotti, G.; Colleoni, S.; Turini, P.; Ponderato, N.; Duchi, R.; Lazzari, G. A cloned horse born to its dam twin-A birth announcement calls for a rethink on the immunological demands of pregnancy. Nature 2003, 424, 635. [CrossRef]

7. Kuwayama, H.; Tanabe, Y.; Wakayama, T.; Kishigami, S. Birth of cloned mice from vaginal smear cells after somatic cell nuclear transfer. Theriogenology 2017, 94, 79-85. [CrossRef] [PubMed]

8. Lee, S.; Zhao, M.; No, J.; Nam, Y.; Im, G.-S.; Hur, T.-Y. Dog cloning with in vivo matured oocytes obtained using electric chemiluminescence immunoassay-predicted ovulation method. PLoS ONE 2017, 12, e0173735. [CrossRef] [PubMed]

9. Liu, Z.; Cai, Y.; Wang, Y.; Nie, Y.; Zhang, C.; Xu, Y.; Zhang, X.; Lu, Y.; Wang, Z.; Poo, M.; et al. Cloning of Macaque Monkeys by Somatic Cell Nuclear Transfer. Cell 2018, 174, 245. [CrossRef]

10. Fan, Z.; Fang, Y.; Sun, M.; Zhao, X. Precise Cell Injection and Extraction Control Based on Microscopic Visual Feedback. IEEE/ASME Trans. Mechatron. 2019, 25, 872-881. [CrossRef] 
11. Huang, H.B.; Sun, D.; Mills, J.K.; Li, W.J.; Cheng, S.H. Visual-Based Impedance Control of Out-of-Plane Cell Injection Systems. IEEE Trans. Autom. Sci. Eng. 2009, 6, 565-571. [CrossRef]

12. Zhang, Y.; Tan, K.K.; Huang, S. Vision-Servo System for Automated Cell Injection. IEEE Trans. Ind. Electron. 2008, 56, 231-238. [CrossRef]

13. Yang, H.; Li, X.; Liu, Y.; Sun, D. Automated Transportation of Biological Cells for Multiple Processing Steps in Cell Surgery. IEEE Trans. Autom. Sci. Eng. 2017, 14, 1712-1721. [CrossRef]

14. Loi, P.; Iuso, D.; Czernik, M.; Ogura, A. A New, Dynamic Era for Somatic Cell Nuclear Transfer? Trends Biotechnol. 2016, 34, 791-797. [CrossRef] [PubMed]

15. Xie, H.; Zhang, H.; Song, J.; Meng, X.; Wen, Y.; Sun, L. High-Precision Automated Micromanipulation and Adhesive Microbonding With Cantilevered Micropipette Probes in the Dynamic Probing Mode. IEEE/ASME Trans. Mechatron. 2018, 23, 1425-1435. [CrossRef]

16. Ichikawa, A.; Tanikawa, T.; Matsukawa, K.; Takahashi, S.; Ohba, K. Fluorescent monitoring using microfluidics chip and development of syringe pump for automation of enucleation to automate cloning. In Proceedings of the IEEE International Conference on Robotics and Automation, Kobe, Japan, 12-17 May 2009; pp. 2231-2236. [CrossRef]

17. Ichikawa, A.; Sakuma, S.; Sugita, M.; Shoda, T.; Tamakoshi, T.; Akagi, S.; Arai, F. On-chip enucleation of an oocyte by untethered microrobots. J. Micromechan. Microeng. 2014, 24, 095004. [CrossRef]

18. Hagiwara, M.; Kawahara, T.; Feng, L.; Yamanishi, Y.; Arai, F. High Precision Magnetically Driven Microtools with Ultrasonic Vibration for Enucleation of Oocytes. In Proceedings of the International Symposium on Micro-NanoMechatronics and Human Science, Nagoya, Japan, 7-10 November 2010; pp. 47-52.

19. Feng, L.; Di, P.; Arai, F. High-precision motion of magnetic microrobot with ultrasonic levitation for 3-D rotation of single oocyte. Int. J. Robot. Res. 2016, 35, 1445-1458. [CrossRef]

20. Inomata, N.; Mizunuma, T.; Yamanishi, Y.; Arai, F. Omnidirectional Actuation of Magnetically Driven Microtool for Cutting of Oocyte in a Chip. J. Microelectromech. Syst. 2011, 20, 383-388. [CrossRef]

21. Feng, L.; Hagiwara, M.; Ichikawa, A.; Arai, F. On-Chip Enucleation of Bovine Oocytes using Microrobot-Assisted Flow-Speed Control. Micromachines 2013, 4, 272-285. [CrossRef]

22. Hagiwara, M.; Kawahara, T.; Yamanishi, Y.; Masuda, T.; Feng, L.; Arai, F. On-chip magnetically actuated robot with ultrasonic vibration for single cell manipulations. Lab Chip 2011, 11, 2049-2054. [CrossRef]

23. Peura, T.; Lewis, I.; Trounson, A. The effect of recipient oocyte volume on nuclear transfer in cattle. Mol. Reprod. Dev. 1998, 50, 185-191. [CrossRef]

24. Wakayama, T.; Yanagimachi, R. Fertilisability and developmental ability of mouse oocytes with reduced amounts of cytoplasm. Zygote 1998, 6, 341-346. [CrossRef]

25. Zhao, C.; Liu, Y.; Sun, M.; Zhao, X. Robotic Cell Rotation Based on Optimal Poking Direction. Micromachines 2018,9 , 141. [CrossRef]

26. Wang, Z.; Feng, C.; Ang, W.T.; Tan, S.Y.M.; Latt, W.T. Autofocusing and Polar Body Detection in Automated Cell Manipulation. IEEE Trans. Biomed. Eng. 2016, 64, 1099-1105. [CrossRef]

27. Zhao, Q.; Wu, M.; Cui, M.; Qin, Y.; Yu, J.; Sun, M.; Zhao, X.; Feng, X. A novel pneumatic micropipette aspiration method using a balance pressure model. Rev. Sci. Instrum. 2013, 84, 123703. [CrossRef]

28. Wang, X.; Zhao, Q.; Wang, L.; Liu, J.; Pu, H.; Xie, S.; Ru, C.; Sun, Y. Effect of Cell Inner Pressure on Deposition Volume in Microinjection. Langmuir 2018, 34, 10287-10292. [CrossRef] [PubMed]

29. Liu, Y.; Wang, X.; Zhao, Q.; Zhao, X.; Sun, M. Robotic Batch Somatic Cell Nuclear Transfer Based on Microfluidic Groove. IEEE Trans. Autom. Sci. Eng. 2020, 17, 2097-2106. [CrossRef]

30. Liu, Y.; Cui, M.; Huang, J.; Sun, M.; Zhao, X.; Zhao, Q. Robotic Micropipette Aspiration for Multiple Cells. Micromachines 2019, 10, 348. [CrossRef]

31. Liu, Y.; Cui, M.; Sun, Y.; Feng, Z.; Bai, Y.; Sun, M.; Zhao, Q.; Zhao, X. Oocyte orientation selection method based on the minimum strain position in the penetration process. J. Appl. Phys. 2019, 125, 154701. [CrossRef]

32. Zhao, Q.; Qiu, J.; Feng, Z.; Du, Y.; Liu, Y.; Zhao, Z.; Sun, M.; Cui, M.; Zhao, X. Robotic Label-free Precise Oocyte Enucleation for Improving Developmental Competence of Cloned Embryos. IEEE Trans. Biomed. Eng. 2020, 1. [CrossRef]

33. Zhao, Q.; Shirinzadeh, B.; Cui, M.; Sun, M.; Liu, Y.; Zhao, X. A novel cell weighing method based on the minimum immobilization pressure for biological applications. J. Appl. Phys. 2015, 118, 044301. [CrossRef]

34. Feng, Z.; Zhao, Q.; Liu, Y.; Sun, M.; Zhao, X.; Cui, M.; Zhao, X. Augmented Reality-Based Precise Oocyte Enucleation. In Proceedings of the IEEE 19th International Conference on Nanotechnology (IEEE-NANO), Macao, China, 22-26 July 2019; pp. 530-534. [CrossRef] 